\title{
The Selection of New Students RSBI Using Fuzzy SAW Based Application
}

\author{
Aeri Rachmad ${ }^{1}$, Muhammad Ali Syakur ${ }^{2}$, Erick Widjaya ${ }^{3}$, Yoga Dwitya Pramudita ${ }^{4}$, Devi \\ Rosa Anamisa ${ }^{5}$, Sigit Susanto Putro ${ }^{6}$, Eka Mala Sari Rochman ${ }^{7}$, Endah Purwanti ${ }^{8}$ \\ \{aery_r@yahoo.com ${ }^{1}$, endah-p-1@fst.unair.ac.id $\left.{ }^{8}\right\}$ \\ Faculty Sains Technology, Airlangga University, Surabaya, Indonesia ${ }^{18}$, Faculty of Engineering, \\ University of Trunojoyo Madura, Indonesia ${ }^{1234567}$
}

\begin{abstract}
RSBI (International School Stubs) is an international school organized by the ministry of education and culture to educate the nation. Selection of entry to become RSBI students is very strict. The number of students who register is so much that it makes it difficult for the school to select it. Accuracy and value to students become one of the determinants to enter into RSBI students. There are two types of admission tests for students in RSBI classes: written tests and practice tests.. In order for the selection process to be fair and no cheating then built an application to select prospective students RSBI using Fuzzy Simple Additive Weighting (SAW) method. From the system test conducted using prospective student data has an accuracy of $95.8 \%$ and $91.7 \%$ of data applicants RSBI prospective students. The results of 7this accuracy are compared with actual student acceptance.
\end{abstract}

Keywords: RSBI, selection, fuzzy simple additive weighting (SAW).

\section{Introduction}

Pedagogy is an exploit that has been projected to supply direction in getting a child's potential to accomplish ends. Because teaching is a process of transferring knowledge, transfer of values and culture and religion. A pupil must comply with all established rules because compliance is a significant element in reaching ends. Admission of new students is the acceptance and selection activities of prospective participants at school. It is nearly connected to the basic skills of academic interest and talent toward the targeted school level.

Junior high school is one of formal education at the level of basic education. Progress in the field of education can be cultivated through the development of potential and talent of students. To develop the potential is done by a good learning process and quality. A quality educational indicator viewed from the human resources as well as the skills needed in his day. Competition in the world of education is only limited in providing quality educational services and improve the quality of graduates, not to seek profit as much [1]. One of the quality measurements is the presence of International School Stubs (RSBI) which is a national standard school that prepares students based on the Indonesian National Standards of Education (SNP) and international standard so that the graduates are expected to take in international competitiveness. The RSBI school make competitiveness for students increases. The number of new admissions at school is increasing quickly, making it hard for the selection process of learners.

ICCSET 2018, October 25-26, Kudus, Indonesia

Copyright () 2018 EAI

DOI 10.4108/eai.24-10-2018.2280568 
Decision Support System is a computer-based system targeted at assisting decision makers in using certain data and good examples to solve unstructured problems, namely finding solutions that require human intuition in making decisions[2]. This decision support system applies to this research so that the selection process of new students can be done appropriately.

This research uses Simple Additive Weighting (SAW) method because it is renowned for its simplicity method. The SAW method is preferred because it sets the weight value for each attribute, followed by a ranking procedure that will select the best option from a number of options. With this method of ranking, the assessment is expected to be more accurate because it is founded on the value of criteria and weight that has been settled so that will generate accurate solutions.

\section{Methodology}

Decision Support System (DSS) concept was first projected in the early 1970s by Michael S. Scott Morton with term Management Decision Systems. The system is a computerbased system intended to assist decision makers by using certain data and good examples to solve unstructured problems[3].

The term DSS refers to a system that utilizes computer support in the decision-making process. To provide a more in-depth understanding, we will describe some definitions of DSS developed by some experts, such as by Man and Watson which provide the following definition, the DSS are an interactive system that helps decision makers through the use of data and decision models for solving problems that are semi-structured and unstructured[4].

\subsection{Data Collection}

The data used is the data of new students who enroll in public junior high school 5 Bangkalan. Variables used in this study is the data selection of student enrollment from 2009-2010 until 2010-2011. The amount of data of prospective students who enroll in the 2009-2010 academic year as many as 52 applicants, prospective students who enroll the academic year 2010-2011 as many as 49 applicants. The criteria factor taken is the Writing Test consist of Indonesian, General Science, Mathematics, Natural Science, Psychotest. While the Practice Test consists of Computers, English, Religion.

\subsection{Simple Additive Weighting (SAW)}

The SAW method is often also known as the weighted summing method[5]. Because the decision maker gives an assessment or weight to each of its alternatives. The basic concept of SAW method is to find the weighted sum of performance ratings on each alternative on all attributes[6]. The SAW method requires the process of normalizing the decision matrix $(\mathrm{X})$ to a scale comparable to all existing alternative ratings[7].

$\mathrm{r}_{\mathrm{ij}}= \begin{cases}\frac{x_{i j}}{\operatorname{Max} x_{i j}} & \text { If } \mathrm{j} \text { is a profits attribute } \\ & \end{cases}$ 
Where $r_{i j}$ is a normalized performance rating of the alternative $A_{i}$ on the attribute $C_{j} ; i=$ $1,2, \ldots, m$ and $j=1,2, \ldots, n$. The preference value for each alternative $\left(V_{i}\right)$ is given as[7]:

$$
\mathrm{V}_{\mathrm{i}}=\sum_{j=1}^{n} \mathrm{~W}_{\mathrm{j}} \mathrm{r}_{\mathrm{ij}}
$$

A larger value of $\mathrm{V}_{\mathrm{i}}$ indicates that $\mathrm{A}_{\mathrm{i}}$ alternatives are preferred. The advantage of the SAW method is to determine the weight value for each attribute, then proceed with a ranking process that will select the best alternative from a number of alternatives[8]. Assessment would be more appropriate because it is based on the criterion value of the preference weight that has been determined. And the calculation of matrix normalization according to the value of the attribute (between the value of benefit and cost). The shortcomings of the SAW method are only applicable to local weighting and the calculation process is performed using both crisp and fuzzy numbers[9].

\subsection{Flowchart System}

A flowchart is a picture in the form of flowchart of the algorithms in a program, which states the direction of the program flow. Flowchart system flow to determine RSBI classroom students using Fuzzy SAW as shown in Figure 1 and Figure 2.

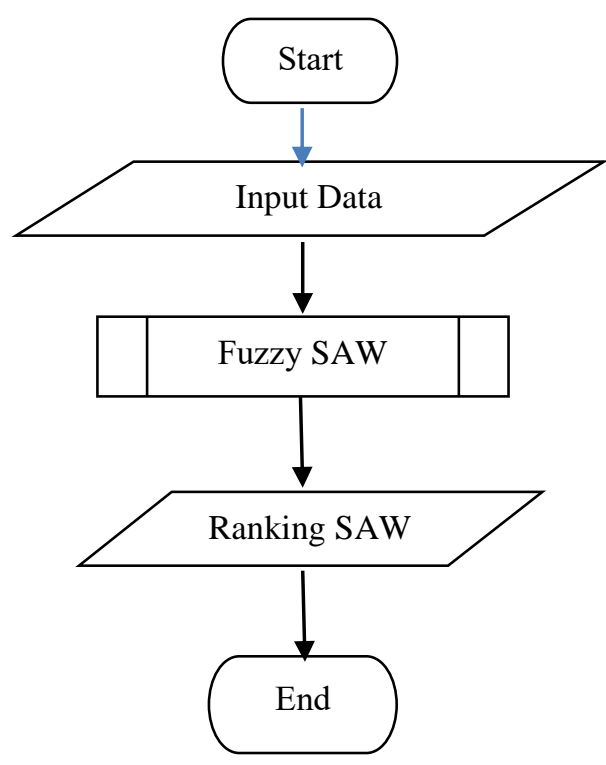

Fig. 1. Overall System Flow. 


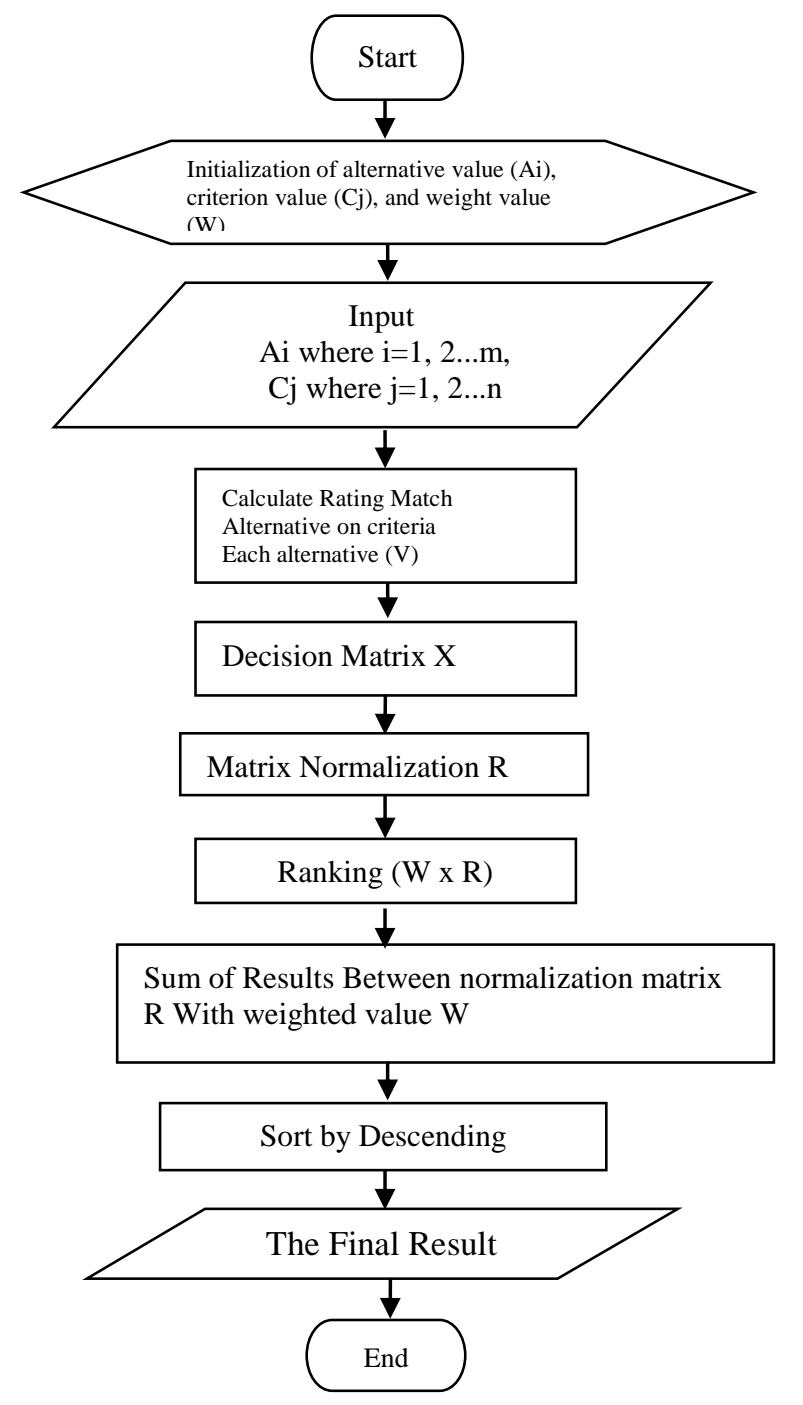

Fig. 2. Simple Additive Weighting (SAW).

\subsection{Output Analysis}

The existing data then fuzzy process to change the membership value between the range 0 to 1 with a range of values greater than 90, 90-76, 75-61, 60-50, and less than 50. Further data is included in the process Simple Additive Weighting (SAW). The SAW method is often also known as the weighted summing method. The basic concept of SAW method is to find the weighted sum of performance ratings on each alternative on all attributes[10]. The SAW method requires the process of normalizing the decision matrix $(\mathrm{X})$ to a scale comparable to all existing alternative ratings[8]. The normalization process used in this system using formula normalization that already exists in SAW method[11]. 
The output generated from this study is an alternative that has the highest value compared with other alternatives. In this study the output is taken from the highest alternative to the lowest alternative for prospective students who have signed up. The alternatives in question are prospective students who enroll in the RSBI class. The final results issued by the program will come from the value of each criterion because in each criterion has different values.

a) Data Entry Criteria and Sub Criteria

In input data, the form that must be filled is the form of process criteria data, as you can see in Figures 3 and 4.

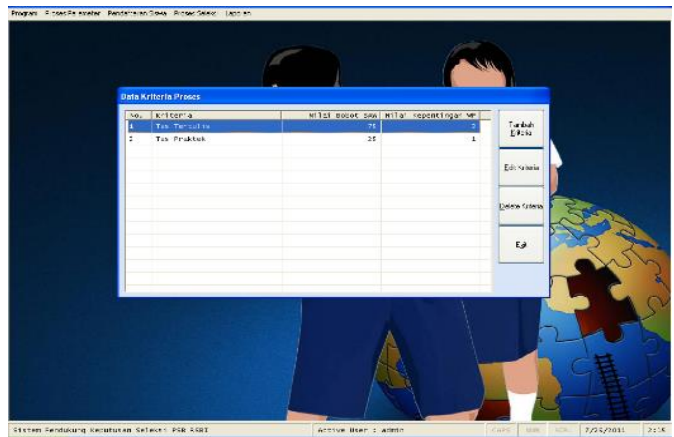

Fig. 3. Criteria data form.

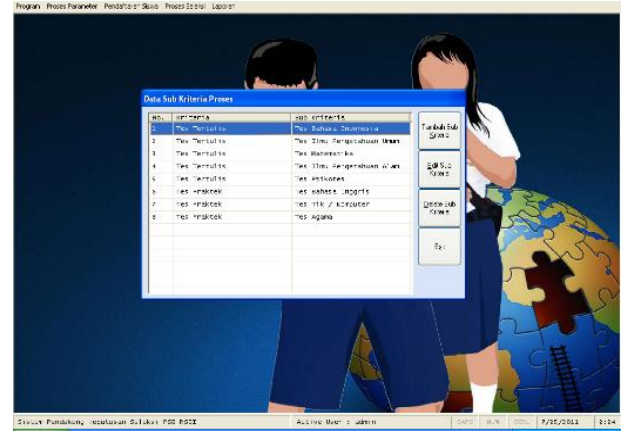

Fig. 4. Sub Criteria data form.

b) Determining the Range of Values and Rating Based on Criteria / Sub Criteria In the process of admission of RSBI class students using Fuzzy SAW method, the form that must be filled in is the criteria/sub criteria rating data form, as you can see in figure 5 and Figure 6 . This form serves to process the criteria/sub criteria, in this case using Fuzzy weighting methods.

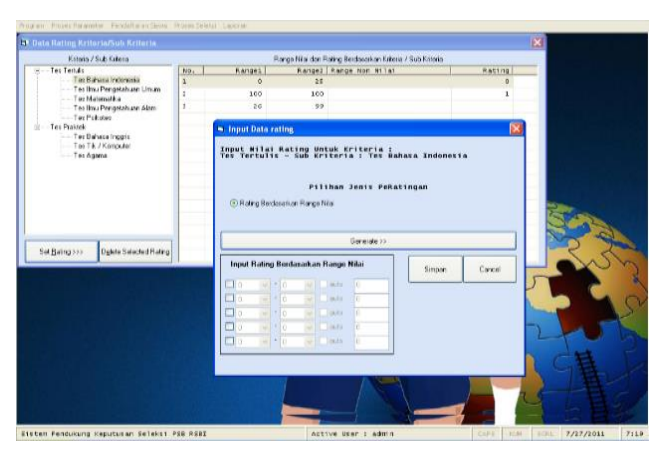

Fig. 5. Rating Sub Criteria Form.

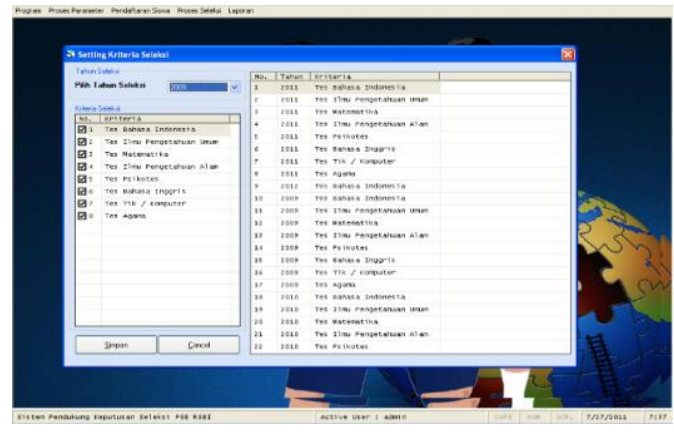

Fig. 6. Setting Sub Criteria Form.

c) Input Student Data and Student Value Data

To input student data and input of student value, before having to do input process criteria, input sub-criteria, and setting criteria / sub-criteria. The filled form is the student data form you can see in figure 7 and figure 8 . 


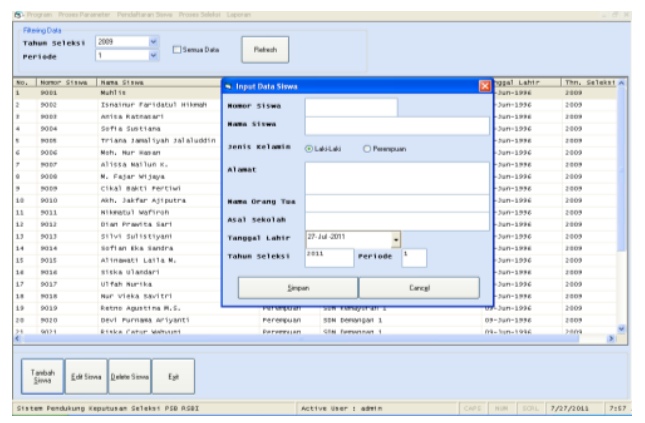

Fig. 7. student data form.

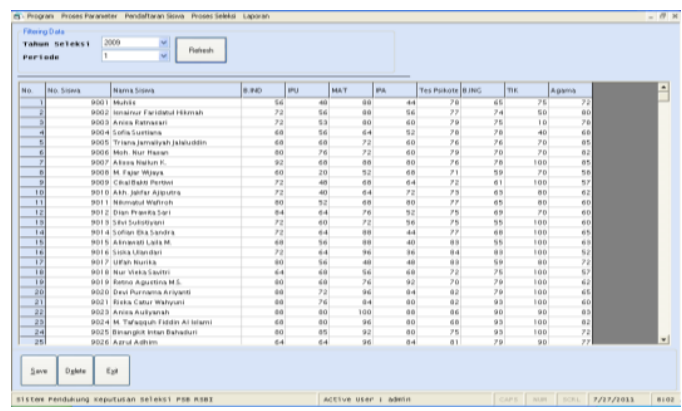

Fig. 8. student value form.

\section{Analysis of Test Results}

Table 1. The results of trials conducted through two scenarios to compare with reality.

\begin{tabular}{cccc}
\hline Scenario & Amount of Data & Criteria & Fuzzy SAW (\%) \\
\hline 1 & 52 & 7 & 95.8 \\
2 & 49 & 8 & 91.7 \\
\hline
\end{tabular}

Table 1 shows the results of the Fuzzy SAW method. In the Fuzzy SAW method, direct data are processed using weights and SAW steps to generate rankings. In the trial scenario 1, for the 2009-2010 school year the data were 52 and who passed the 24 selections according to the school ceiling using 7 criteria, with the accuracy of $95.8 \%$. In the trial scenario 2 , for the 2010-2011 school year the data were 49 and who passed the selection 24 according to the school ceiling using 8 criteria, with an accuracy of $91.7 \%$.

\section{Conclusion}

This scheme can be applied as a solution to determine new students RSBI class. From several scenarios tested, the output of the system shows that using the Fuzzy SAW method has more than $90 \%$. This is because SAW has the basic concept of getting a weighted amount of performance evaluations on each alternative on all properties.

\section{Acknowledgment}

We would like to thank the head of the Informatics Engineering Department, Faculty of Engineering, the University of Trunojoyo Madura who has provided an opportunity to publish the results of this research. We also convey to the colleagues of informatics engineering lecturers and all the residents of Multimedia and Networking Labs who have assisted the completion of this research.

\section{References}

[1] S. Ma'arif, "RINTISAN SEKOLAH BERSTANDAR INTERNASIONAL: Antara Cita \& Fakta," Walisongo, pp. 399-428, 2011. 
[2] M. N. S. A. H. S. G, "A quantitative discussion on the assessment of power supplytechnologies: DEA (Data Envelopment Analysis) and SAW (Simple Additive Weighting) as complementary methods for the "Grammar,"' Energy, vol. 64, pp. 640-647, 2014.

[3] F. F. e. A, "Decission Support System," Springer Int. Publ., p. 31, 2017.

[4] D. P. I. Kaliszewski, "Simple Additive Weighting - a meta model for Multiple Criteria Decision Analysis methods," Expert Syst. Appl., 2016.

[5] F. S. A. A. J. M. A. G. F. S. J. Seyedmohammadia, "Application of SAW, TOPSIS and fuzzy TOPSIS models in cultivation priority planning for maize, rapeseed and soybean crops," Geoderma, vol. 310, pp. 178-190, 2018.

[6] Y.-J. Wang, "A fuzzy multi-criteria decision-making model based on simple additive weighting method and relative preference relation," Appl. Soft Comput., vol. 30, pp. 412-420, 2015.

[7] Y. W. Peng Wang, Zhouquan Zhu, "A novel hybrid MCDM model combining the SAW, TOPSIS and GRA methods based on experimental design," Inf. Sci. (Ny)., 2016.

[8] T.-Y. Chen, "Comparative analysis of SAW and TOPSIS based on interval-valued fuzzy sets: Discussions on score functions and weight constraints," Expert Syst. Appl., vol. 39, pp. 1848-1861, 2012.

[9] D. K. E. Roszkowska, "The fuzzy saw and fuzzy TOPSIS procedures based on ordered fuzzy numbers," Inf. Sci. (Ny)., 2016.

[10] A. C. Alireza Arab Ameri, Hamid Reza Pourghasemi, "Erodibility prioritization of subwatersheds using morphometric parameters analysis and its mapping: A comparison among TOPSIS, VIKOR, SAW, and CF multi-criteria decision making models," Sci. Total Environ., vol. 613-614, pp. 1385-1400, 2018.

[11] C.-Y. S. Shuo-Yan Chou a, Yao-Hui Chang, "A fuzzy simple additive weighting system under group decision-making for facility location selection with objective/subjective attributes," Eur. J. Oper. Res., vol. 189, pp. 132-145, 2008. 